\title{
Reducción de los Niveles de Estrés Docente y los Días de Baja Laboral por Enfermedad en Profesores de Educación Secundaria Obligatoria a través de un Programa de Entrenamiento en Mindfulness
}

\section{Reducing Levels of Teacher Stress and the Days of Sick Leave in Secondary School Teachers through a Mindfulness Training Programme}

\author{
Israel Mañas Mañas, Clemente Franco Justo y Eduardo Justo Martínez \\ Universidad de Almería, España
}

\begin{abstract}
Resumen. El objetivo del presente estudio es examinar la eficacia de un programa de entrenamiento en mindfulness en reducir los niveles de estrés docente y los días de baja laboral por enfermedad en un grupo de profesores de Educación Secundaria Obligatoria (ESO). La muestra estuvo constituida por 31 profesores de ESO, los cuales fueron aleatoriamente asignados; 16 formaron el grupo experimental y los 15 restantes el grupo control. Los niveles de estrés docente fueron evaluados mediante la Escala de Estrés Docente (ED-6) antes y después de la aplicación del programa. Los análisis estadísticos muestran la reducción significativa de los niveles de estrés docente y de los días de baja laboral por enfermedad, así como también en tres de las seis dimensiones que evalúan la Escala ED-6 (presiones, desmotivación y mal afrontamiento) en el grupo experimental en comparación con el grupo control.

Palabras clave: estrés docente, Educación Secundaria Obligatoria, bajas laborales, conciencia plena.
\end{abstract}

\begin{abstract}
The purpose of the current study is to examine the efficacy of a mindfulness training program to reduce the levels of teacher stress and the days of sick leave taken by a group of teachers. The sample comprised 31 teachers of Secondary School Education, who were randomly allocated; 16 of them formed the experimental group and the 15 remaining the control group. The levels of teacher stress were measured by the Teacher Stress Scale (ED6) before and after the application of the programme. Statistical analysis shows the significant reduction of levels of teacher stress and the number of days of sick leave, as well as in three dimensions of the ED-6 (pressure, demotivation, and coping poorly) in the experimental group compared with the control group.

Keywords: teacher stress, Secondary School Education, sick leave, mindfulness.
\end{abstract}

El estrés es una de las cargas emocionales más fuertes que sufre la humanidad hoy en día (Peiró, Zurriaga y González, 2002). Sus principales sínto-

La correspondencia sobre este artículo debe enviarse al primer autor al Dpto. Personalidad, Evaluación y Tratamientos Psicológicos, Facultad de Psicología, Universidad de Almería. La Cañada de San Urbano. Ctra. de Sacramento s/n.04130 Almería. E-mail: imanas@ual.es mas son el nerviosismo, la irritabilidad, las contracturas musculares y el agotamiento físico y psicológico (Turner, Wheaton y LLoyd, 1995). Además, se considera que es la principal causa de gran parte de las enfermedades en el mundo occidental (Sandín, 2003), siendo su principal consecuencia la falta de equilibrio en el organismo como respuesta a las 
influencias del medio ambiente (Cockerham, 2001). El estrés psicosocial es considerado como un factor que predispone al desarrollo de diversas enfermedades en el ser humano, cuyos efectos impactan sobre su calidad de vida y sobre su salud (Molina, Gutiérrez, Hernández y Contreras, 2008), por lo que vivir situaciones de estrés suele asociarse a una reducción de conductas de salud y a un incremento de conductas nocivas para ésta (Guerrero, 2003). Ponce, Bulnes, Aliaga, Atalaya y Huertas (2005) consideran que el estrés laboral es un factor importante que interacciona con otras variables psicológicas, biológicas y sociales dando lugar a la aparición de nuevas enfermedades tanto físicas como mentales, de tal modo, que está relacionado con el $50 \%$ de las bajas laborales que tienen lugar. El impacto negativo que el estrés laboral ejerce sobre la salud física y psicológica de las personas que lo padecen, se traduce en trastornos cardiovasculares, deterioro del sistema inmunológico, ansiedad, depresión, incremento del consumo de drogas, etc. (McEwen, 2002).

En el contexto de la docencia, diversos factores como la falta de disciplina, los problemas de comportamiento, la apatía, el bajo rendimiento y la falta de motivación por el aprendizaje de los alumnos, se han convertido en importantes fuentes de estrés y ansiedad para el profesorado que repercuten de forma negativa tanto en su rendimiento laboral como en su salud psicofísica (Extremera y Fernández-Berrocal, 2004). La presión a la que se ven sometidos los docentes desde diversos frentes, deriva en la actualidad en la aparición creciente de patologías tanto de carácter físico como psicológico, de tal modo que cada vez la comunidad educativa tiende a prestar mayor atención y preocupación por un problema que afecta tanto a la salud del profesorado como a la calidad de la enseñanza (Sugai y Horner, 2001; Troman, 2000; Woods y Carlyle, 2002). En este sentido, Ponce et al. (2005) señalan que cuando el estrés intenso se mantiene a lo largo del tiempo suelen aparecer en los docentes sentimientos negativos, actitud de cinismo, falta de implicación con los alumnos, absentismo laboral, ansiedad, depresión, irritabilidad, descenso en la autoestima, insomnio, hipertensión, úlceras, trastornos coronarios, consumo de drogas y alcohol, etc.
Bermejo y Prieto (2006) hallaron, en una muestra de profesores de Educación Secundaria Obligatoria (ESO), que la baja tolerancia a la frustración juega un papel fundamental en los niveles de estrés, depresión y sintomatología física en esta población. Por su parte, Moriana y Herruzo (2005) han constatado que ciertos trastornos psicopatológicos que padecen los profesores de ESO, principalmente el estrés y la ansiedad, están estrechamente relacionados con los factores que forman el síndrome de burnout, y que éste a su vez, supone un excelente predictor de las bajas laborales de tipo psiquiátrico. Por otro lado, Moreno, Garrosa y González-Romá (2000) encontraron que los profesores que se abstienen de utilizar estrategias de afrontamiento poco adaptativas, tales como la evitación, el distanciamiento o la negación, presentan menores niveles de estrés y de desgaste profesional. Por último, Riquelme, Buendía y Rodríguez (1993) han señalado que, ante una determinada situación estresante, las estrategias centradas en el autocontrol y la exposición son más eficaces que aquellas que se basan en la huida y/o evitación de la situación generadora de estrés.

Desafortunadamente, la formación académica del profesorado de ESO no incluye ningún tipo de preparación psicológica, ni herramienta alguna de autoconocimiento personal, por lo que los docentes suelen carecer de los recursos y habilidades necesarios para poder hacer frente a las exigencias y a las demandas que su labor docente les plantea día a día (Anadón, 2005; Bisquerra, 2005). En este sentido, Moriana y Herruzo (2004) establecen que una mayor autoconsciencia y un mayor nivel de autocontrol y autoeficacia, pueden ejercen un factor protector frente al estrés que sufren los docentes. En concordancia con este planteamiento, Shapiro y Schwartz (2000) consideran que el desarrollo de la capacidad de atención produce mejoras sobre el funcionamiento del sistema de autorregulación de la conducta encargado de mantener el equilibrio y la adaptación del organismo.

En este contexto, la meditación es una técnica que podría resultar especialmente útil. Actualmente, la meditación se está empleando bajo la rúbrica "mindfulness" o "conciencia plena" (e.g., véase Baer, 2003; Kabat-Zinn, 1990, 2003a, 
2003b; Mañas, 2009; Miró, 2006; Simón, 2006; Vallejo, 2006), principalmente en el ámbito de psicología y la medicina, para el tratamiento de múltiples enfermedades médicas y trastornos psicológicos. La psicología contemporánea emplea al mindfulness para incrementar la consciencia y responder habilidosamente a los procesos mentales que contribuyen al desarrollo de malestar emocional y comportamientos desadaptativos (Bishop et al., 2004). Hoy día, son numerosos los estudios que muestran la eficacia y las múltiples aplicaciones de las técnicas, programas y terapias psicológicas basadas en mindfulness (e.g., Arias, Steinberg, Banga y Trestman, 2006; Baer, 2003; Brown, Ryan y Creswell, 2007; Chiesa y Serreti, 2009; Germer, Siegel y Fulton, 2005; Greeson, 2009; Irving, Dobkin y Park, 2009; Segal, Williams y Teasdale, 2002). Kabat-Zinn (1990) describió al mindfulness como la capacidad de llevar la atención a las experiencias que se están experimentando en el momento presente, de un modo particular, aceptándolas y sin juzgar. De forma más general, mindfulness ha sido descrito como una clase de conciencia centrada en el presente, no elaborativa ni condenatoria, en la cual cada pensamiento, sentimiento o sensación que surge en el campo atencional es reconocido y aceptado tal y como es (Kabat-Zinn, 2003a, 2003b; Shapiro y Schwartz, 2000; Segal et al., 2002).

La meditación es una técnica cuyos componentes principales son la concentración y la atención consciente de la mente. De hecho, la práctica regular de la meditación permite alcanzar un estado en el que el cuerpo está relajado, la mente se encuentra tranquila y concentrada, y en el que podemos percibir las sensaciones del momento presente (Thera, 2003). Así pues, la práctica de la meditación radica en centrar o focalizar la atención, ya que para meditar debemos concentrarnos en un estímulo (e.g., respiración, imagen, sonido, etc.) y hacer de éste nuestro objeto de meditación, de tal manera que manteniendo nuestra atención focalizada en ese estímulo conseguimos distanciarnos de nuestros pensamientos, obsesiones y preocupaciones (Harrison, 2004). La meditación nos lleva a un estado en el que se es consciente de la realidad del momento presente, aceptando y reconociendo lo que existe en el aquí y el ahora, pero sin quedar atrapado en los pensamientos o en las reacciones emocionales que la situación provoca (Kabat-Zinn, 2003b). Es decir, se aprende a ser más conscientes de los pensamientos y las emociones que tienen lugar en cada momento, cambiando nuestra forma de relacionarnos y de reaccionar ante ellos. De este modo, los pensamientos y los sentimientos pasan a contemplarse como eventos mentales transitorios que no tienen porque reflejar la realidad del momento presente.

Diversos estudios han demostrado que la práctica regular y continua de la meditación produce diferentes efectos beneficiosos sobre el organismo, algunos de ellos relacionados con el estrés, como por ejemplo: disminuciones en los niveles de cortisol (Maclean et al., 1997); incrementos de los niveles de melatonina y serotonina (Solberg et al., 2004); una disminución del metabolismo incluso mayor que la producida durante el sueño (Benson, Malhotra, Goldman, Jacobs y Hopkins, 1990); reducción de los índices de lactato en sangre, que es un marcador del estrés y de la ansiedad (Solberg et al., 2000); reducción de la tensión arterial (Barnes, Treiber y Davis, 2001); mejora significativa del insomnio (Jacobs, Benson y Friedman, 1993); reducciones de los niveles de nerviosismo, preocupación y malestar emocional, e incrementos de los niveles de relajación muscular, tranquilidad emocional y del grado de bienestar general (Mañas, Luciano y Sánchez, 2008; Mañas, Sánchez y Luciano, 2008), entre otros. Por todo ello, el Instituto Nacional de Salud de los Estados Unidos, viene recomendando desde hace más de una década el uso de la meditación como tratamiento eficaz y útil en diversos trastornos psicopatológicos y médicos como la ansiedad, el insomnio, los dolores de cabeza, los ataques de pánico y el dolor crónico (Cerlin y Lee, 1997).

Según lo expuesto anteriormente, la hipótesis de la presente investigación consiste en que un grupo de docentes de ESO participantes en un programa de entrenamiento en mindfulness van a experimentar una reducción significativa en sus niveles de estrés docente, y consiguientemente, en el número de días de baja laboral por enfermedad, en comparación con otro grupo de docentes de ESO no participantes en el programa de entrenamiento en mindfulness. 


\section{Método}

\section{Participantes}

La muestra estuvo constituida por 31 profesores de ESO (8 hombres y 23 mujeres) pertenecientes a centros públicos de la provincia de Granada (España). Las edades de los mismos oscilaron entre los 26 y los 58 años $(M=38,67 ; D T=10,34)$. Dieciséis participantes fueron asignados al grupo experimental (4 hombres y 12 mujeres), y los 15 restantes al grupo control (4 hombres y 11 mujeres). La asignación de los sujetos a uno u otro grupo se realizó al azar de manera aleatoria, controlándose la variable sexo para que hubiese aproximadamente el mismo número de hombres y de mujeres en ambos grupos.

\section{Instrumentos}

Para la evaluación de los niveles de estrés docente, se empleó la Escala de Estrés Docente (ED-6) (Gutiérrez, Morán y Sanz, 2005), que se compone de 77 ítems agrupados en seis dimensiones: ansiedad, depresión, presiones, creencias desadaptativas, desmotivación y mal afrontamiento. Sumando las puntuaciones en las seis variables anteriores, se obtiene la puntuación global de estrés docente. Entre 126 y 147 indicaría un nivel muy bajo de estrés docente; entre 148 y 164 el nivel es bajo; entre 165 y 189 normal; entre 190 y 212 moderado; y puntuaciones superiores a 213 indican un nivel alto. Cada ítem hace referencia a una afirmación ante la que el docente debe de mostrar su grado de desacuerdo o acuerdo mediante una escala tipo Likert de 1 a 5 puntos (de $1=$ total desacuerdo a $5=$ total acuerdo). Esta escala muestra unas propiedades psicométricas aceptables, teniendo en cuenta que el Coeficiente Alfa de todos los factores se encuentra entre .74 y .89 , siendo la fiabilidad total de la escala de .93 .

\section{Diseño}

Para analizar los efectos del programa de meditación (variable independiente) sobre los niveles de estrés y los días de baja laboral por enfermedad de los docentes (variables dependientes), se utilizó un diseño de comparación de grupos con medidas pretest-postest, con un grupo experimental y un grupo control.

\section{Procedimiento}

En primera instancia se procedió a obtener la muestra del estudio. Para ello, se ofertó un curso de formación para profesores de ESO titulado "Prevención y tratamiento del estrés", al que se inscribieron un total de 31 profesores. Seguidamente, se realizó la asignación al azar de los sujetos a los grupos control y experimental, controlándose la variable sexo para que hubiese el mismo número de hombres y de mujeres en ambos grupos, ya que las profesoras se ven más afectadas por el estrés docente que los profesores (véase Santiago, Otero, Castro y Villardefrancos, 2008).

Una vez obtenida la muestra, se procedió a la evaluación pretest de todos los participantes en la investigación, para lo cual se les pidió que cumplimentasen la Escala ED-6 con el objetivo de conocer los niveles de estrés docente de partida de cada uno de ellos. También se registró el número de días de baja laboral por enfermedad, de todos los participantes, durante el curso académico anterior (2008/2009). Para el cómputo de dichos días no se tuvieron en cuenta las bajas laborales por maternidad, por accidente, ni por enfermedad de un familiar. Posteriormente, a los sujetos del grupo control se les comunicó que por razones de espacio el grupo tenía que ser dividido y que el curso se impartiría en dos turnos, pasando ellos a formar parte del segundo turno

Una vez obtenida la puntuación pretest de las variables del estudio, se inició la aplicación del programa de entrenamiento en mindfulness en el grupo experimental. El programa tuvo una duración total de 15 horas, que fueron impartidas a lo largo de 10 semanas con una duración aproximada de una hora y media cada sesión. El programa de entrenamiento consistió, principalmente, en el aprendizaje y práctica diaria de una técnica de meditación mindfulness llamada Meditación Fluir (Franco, 2007, 2009a). El objetivo de esta técnica no consiste en tratar de controlar los pensamientos, sensaciones o sentimientos, 
ni modificarlos o cambiarlos por otros, sino todo lo contrario, dejarlos libres, aceptando cualquier evento privado que pueda aparecer o surgir de forma espontánea.

La Meditación Fluir consiste en tomar conciencia de nuestra respiración mientras se repite de forma simultánea una palabra o mantra. Para ello, se forma una palabra aguda de tres sílabas que debe carecer de significado, y se va repitiendo mentalmente de forma suave y sin esfuerzo, mientras se dirige la atención a la zona del abdomen para sentir como entra y sale de forma natural el aire al respirar. De este modo, la mente se aquieta alcanzando un estado de serenidad mental y de elevada atención y concentración, disminuyendo su actividad automática e involuntaria (véase Holen y Halvor, 2007). Lo único que tenemos que hacer es darnos cuenta de forma pasiva de que estamos respirando y de cómo estamos respirando, pero sin tratar de influir o modificar la respiración, ya que dejamos que ésta tenga lugar de forma natural y espontánea. Asimismo, no hay que esforzarse por tratar de retener y repetir mentalmente la palabra, por lo que no hay que preocuparse si ésta se desvanece y es sustituida por pensamientos e imágenes, ya que se trata de mantener una concentración pasiva. Darnos cuenta y mantener una concentración pasiva significa tomar consciencia de nuestra propia experiencia sin reaccionar a ella ni elaborar juicios, manteniendo una actitud de aceptación y apertura a las experiencias que se van sucediendo momento a momento.

A los participantes se les instruye a que cuando su atención se desvié del objeto de meditación (la respiración y el mantra), no se recriminen por ello, que tomen consciencia del estímulo que ha capturado su atención (pensamiento, imagen, sensación corporal, sonido, etc.) y que seguidamente vuelvan a redirigir el foco de la atención hacia el objeto de meditación. Se enfatiza el mantenimiento de una actitud de paciencia, confianza y persistencia durante el proceso. Por tanto, algunos de los procesos cognitivos básicos implicados en la Meditación Fluir -focalización, mantenimiento y redirección de la atención-, y algunos de sus componentes y aspectos actitudinales -no juzgar, paciencia, confianza, no esforzarse, aceptación, etc.- (para más detalle véase Franco, 2009a) son los mismos procesos, componentes y aspectos actitudinales inherentes al mindfulness (véase Kabat-Zinn, 1990, 2003a, 2003b).

Además del aprendizaje de esta técnica de meditación, el programa de entrenamiento es completado con la presentación y discusión durante las sesiones de diversas metáforas y ejercicios utilizados en la Terapia de Aceptación y Compromiso (Hayes, Strosahl y Wilson, 1999; Wilson y Luciano, 2002), junto con relatos de la tradición Zen (Deshimaru, 2006) y de la meditación Vipassana (Hart, 1994). A través del empleo de las metáforas y ejercicios de la Terapia de Aceptación y Compromiso se pretende reducir la fusión o rigidez cognitiva y aumentar la flexibilidad cognitiva, romper la literalidad de las palabras, generar una desesperanza creativa, desarrollar el yo como contexto o la toma de perspectiva, abandonar el control de los eventos privados (pensamientos, sentimientos, sensaciones corporales, etc.) y promover la aceptación de los mismos (para más detalle véase Eifert y Forsyth, 2005; Eifert et al., 2009; Hayes y Stroshal, 2004; Hayes et al., 1999). Por su parte, y de forma conjunta, con la presentación de los relatos se busca acentuar y reforzar la asunción de cómo, en lo que respecta a los eventos privados molestos y desagradables, el intento de control, en muchas ocasiones, lo único que hace es cronificar y agravar el malestar psicológico producido por dichos eventos privados (véase Purdon, 1999; Rassin, Merckelbach y Muris, 2000; Abramowit, Tolin y Street, 2001), y que por tanto, la mejor opción ante ellos es la de dejarlos fluir libremente. Por último, otra técnica de meditación mindfulness que compone el programa de entrenamiento es la práctica de la exploración corporal o body-scan (véase Kabat-Zinn, 1990, 2003b).

La estructura de las sesiones del programa de entrenamiento fue la siguiente: a) comentarios de los participantes sobre su práctica de los ejercicios de mindfulness durante la semana (dudas, experiencias, dificultades, contratiempos, etc.); b) realización del ejercicio de body-scan durante 10 minutos; c) presentación de las diversas metáforas y ejercicios correspondientes a cada sesión; y d) práctica de conciencia plena atendiendo a la respiración durante 2030 minutos. La Tabla 1 contiene los principales ejercicios y metáforas empleados en cada una de las sesiones. 
Tabla 1. Ejercicios y metáforas del programa de entrenamiento en mindfulness

\begin{tabular}{|c|c|c|}
\hline Sesión $n^{\circ}$ & Ejercicios & Metáforas \\
\hline 1 & $\begin{array}{l}\text { * Presentación de todos los miembros del grupo } \\
\text { * Exposición de los motivos para participar en el curso } \\
\text { de meditación y sus expectativas } \\
\text { * Exposición de las condiciones previas necesarias para comenzar } \\
\text { a meditar y diversos aspectos a tener en cuenta en la práctica de } \\
\text { la meditación } \\
\text { * Explicación de la práctica de la meditación fluir } \\
\text { * Realización de la primera práctica de meditación }\end{array}$ & \\
\hline 2 & $\begin{array}{l}\text { * Ejercicio de tratar de no pestañear durante un minuto } \\
\text { * Búsqueda de analogías entre el ejercicio anterior y el control de } \\
\text { eventos privados } \\
\text { * Práctica de meditación }\end{array}$ & Metáfora del bambú \\
\hline 3 & $\begin{array}{l}\text { * Ejercicio de observación de sensaciones en la zona de la cara } \\
\text { * Práctica de meditación }\end{array}$ & $\begin{array}{l}\text { Metáfora del río, metáfora del vaso sucio, metáfora de la ope- } \\
\text { ración, y metáfora del parto con dolor }\end{array}$ \\
\hline 4 & $\begin{array}{l}\text { * Ejercicio de observación de sensaciones en la zona del pecho } \\
\text { y del abdomen } \\
\text { * Práctica de meditación }\end{array}$ & Metáfora del mono y el plátano \\
\hline 5 & $\begin{array}{l}\text { * Ejercicio de observación de sensaciones en la zona de la espalda } \\
\text { * Ejercicio de recordar palabras } \\
\text { * Ejercicio de observación de pensamientos } \\
\text { * Ejercicio ¿cuáles son los números? y ¿cómo se llama tu madre? } \\
\text { * Práctica de meditación }\end{array}$ & Metáfora de los dos monjes \\
\hline 6 & $\begin{array}{l}\text { * Ejercicio de observar las sensaciones en los brazos } \\
\text { * Ejercicio de contar pensamientos } \\
\text { * Práctica de meditación }\end{array}$ & Historia de las dos sortijas \\
\hline 7 & $\begin{array}{l}\text { * Ejercicio de observar las sensaciones en las piernas } \\
\text { * Práctica de meditación }\end{array}$ & Metáfora de la radio encendida \\
\hline 8 & $\begin{array}{l}\text { * Ejercicio de observación de sensaciones por todo el cuerpo } \\
\text { * Ejercicio para analizar los "debo de...", y los "debería de...." }\end{array}$ & \\
\hline 9 & $\begin{array}{l}\text { * Ejercicio de aceptación de eventos privados molestos e incómodos } \\
\text { * Ejercicio de decir sí y hacer no y decir no y hacer sí } \\
\text { * Práctica de meditación }\end{array}$ & $\begin{array}{l}\text { Metáfora del ordenador, metáfora del polígrafo, la historia de } \\
\text { miaou }\end{array}$ \\
\hline 10 & * Práctica de meditación & Metáfora de los indios que recogen leña \\
\hline
\end{tabular}

Para garantizar y facilitar, en la medida de lo posible, la implementación y seguimiento del programa, los sujetos fueron instruidos a realizar diariamente en casa el ejercicio de body-scan durante 10 minutos y la práctica de conciencia plena sobre la respiración en la zona del abdomen durante 30 minutos. Además, cada participante debía completar a lo largo de la semana una hoja de autorregistro en la que diariamente debía anotar si había practicado los ejercicios preescritos de body-scan y de conciencia plena de la respiración. Los participantes entregaban semanalmente el autorregistro de su práctica diaria cuando acudían a las sesiones. El grado medio de cumplimiento del ejercicio de body-scan fue del $74 \%$, y del ejercicio de conciencia plena de la respiración $81 \%$.

Una vez finalizada la intervención o aplicación del programa de entrenamiento en el grupo experimental, se procedió a evaluar nuevamente los niveles de estrés docente y a registrar los días de baja laboral por enfermedad durante el curso académico 2009/2010 en ambos grupos (evaluación postest). Para ello, se solicitó a todos los participantes que cumplimentasen de nuevo la Escala ED-6 y se registraron los días de baja laboral por enfermedad durante el curso académico 2009/2010. Posterior- 
mente, todos los participantes en el estudio fueron informados del objetivo de la investigación, y se les solicitó su consentimiento por escrito para poder hacer uso de los datos obtenidos manteniendo y garantizando la confidencialidad y el anonimato. Por último, se procedió a impartir el curso de formación a los profesores del grupo control, tal y como se les había informado previamente.

\section{Resultados}

En primer lugar, se hallaron las puntuaciones medias y las desviaciones típicas para cada una de las variables del estudio correspondientes a los grupos control y experimental en las fases pretest y postest (véase Tabla 2).

Para analizar la existencia de diferencias estadísticamente significativas en las medidas pretest y en tres de la dimensiones relativas a estrés docente: la más significativa en la dimensión desmotivación $(z=2.87, p<.01)$, le sigue la dimensión mal afrontamiento $(z=2.64, p=.01)$, y por último, la dimensión presiones $(z=2.09, p<.05)$. Las puntuaciones en el resto de las dimensiones (ansiedad, depresión y creencias desadaptativas) no difieren entre los grupos tras la intervención.

Para analizar la existencia de diferencias estadísticamente significativas y el grado de incidencia de la intervención en cada una de las variables y en las dimensiones relativas a estrés docente, se compararon las medidas postest con las pretest utilizando el estadístico de contraste no paramétrico de Wilcoxon para muestras relacionadas (véase Tabla 4). La Tabla 4 muestra la no existencia de diferencias significativas en ninguna de las variables en el grupo control. Sin embargo, en el grupo experimental se observan diferencias significativas en las variables días de

Tabla 2. Medias y desviaciones típicas pretest y postest correspondientes a los grupos control y experimental para las variables días de baja laboral, estrés docente y para cada dimensión relativa a estrés docente

\begin{tabular}{|c|c|c|c|c|c|c|c|c|}
\hline & \multicolumn{4}{|c|}{ Pretest } & \multicolumn{4}{|c|}{ Postest } \\
\hline & \multicolumn{2}{|c|}{ Control } & \multicolumn{2}{|c|}{ Experimental } & \multicolumn{2}{|c|}{ Control } & \multicolumn{2}{|c|}{ Experimental } \\
\hline & $M$ & $D T$ & $M$ & $D T$ & $M$ & $D T$ & $M$ & $D T$ \\
\hline Días de baja laboral & 7.53 & 7.78 & 8.25 & 9.32 & 9.60 & 9.92 & 2.19 & 4.21 \\
\hline Estrés docente & 183.73 & 34.64 & 189.00 & 44.74 & 182.01 & 32.71 & 158.44 & 25.81 \\
\hline Ansiedad & 44.47 & 16.48 & 47.25 & 18.66 & 42.93 & 16.30 & 37.81 & 13.28 \\
\hline Depresión & 18.27 & 7.68 & 21.94 & 10.69 & 17.87 & 6.70 & 15.69 & 5.35 \\
\hline Creencias desadap. & 31.20 & 5.44 & 32.81 & 7.08 & 32.07 & 5.48 & 29.88 & 6.35 \\
\hline Presiones & 27.60 & 4.57 & 25.81 & 6.84 & 26.60 & 5.61 & 22.69 & 4.78 \\
\hline Desmotivación & 32.33 & 4.76 & 31.81 & 6.39 & 31.73 & 5.88 & 26.63 & 3.86 \\
\hline Mal afrontamiento & 30.93 & 4.97 & 29.69 & 4.96 & 30.53 & 5.44 & 25.63 & 4.99 \\
\hline
\end{tabular}

postest entre ambos grupos, se utilizó el estadístico de contraste no paramétrico $U$ de Mann-Whitney para muestras independientes, ya que los datos no se ajustaban a la distribución normal de probabilidades (véase Tabla 3). La Tabla 3 muestra la no existencia de diferencias estadísticamente significativas entre ambos grupos en las medidas pretest en ninguna de las variables. Es decir, no existían diferencias entre los grupos en las variables analizadas antes de la intervención. Sin embargo, se observan diferencias estadísticamente significativas entre ambos grupos en las medidas postest, tanto en la variable días de baja laboral $(z=2.73, p=.01)$, como en la variable nivel de estrés docente $(z=2.23, p<.05)$, así como
Tabla 3. Prueba $U$ de Mann-Whitney para muestras independientes de las diferencias pretest y postest entre el grupo control y experimental, para las variables días de baja laboral, estrés docente y para cada dimensión relativa a estrés docente

\begin{tabular}{lllll}
\hline & \multicolumn{2}{c}{ Pretest } & \multicolumn{2}{c}{ Postest } \\
\hline Variable & $z$ & $p$ & $z$ & $p$ \\
\hline Días baja laboral & .23 & .819 & 2.73 & $.010^{* *}$ \\
Estrés docente & .36 & .718 & 2.23 & $.033^{* * *}$ \\
Ansiedad & .43 & .664 & .96 & .344 \\
Depresión & .09 & .284 & 1.03 & .324 \\
Creencias desadap. & .70 & .485 & 1.02 & .314 \\
Presiones & .84 & .403 & 2.09 & $.045^{* * *}$ \\
Desmotivación & .25 & .800 & 2.87 & $.007^{*}$ \\
Mal afrontamiento & .69 & .491 & 2.64 & $.010^{* *}$ \\
\hline
\end{tabular}

Nota: ${ }^{*} p<.01 ; * * p=.01 ; * * *<.05$ 
Tabla 4. Prueba de Wilcoxon para muestras relacionadas de las diferencias postes-pretest en el grupo control y experimental, y $d$ de Cohen para las variables días de baja laboral, estrés docente y para cada dimensión relativa a estrés docente

\begin{tabular}{|c|c|c|c|c|c|c|}
\hline \multirow[b]{2}{*}{ Variable } & \multicolumn{3}{|c|}{ Control } & \multicolumn{3}{|c|}{ Experimental } \\
\hline & $z$ & $p$ & $d$ & $z$ & $p$ & $d$ \\
\hline Días baja laboral & 1.57 & .13 & .23 & 3.68 & $.002 * *$ & .84 \\
\hline Estrés docente & 2.22 & .14 & .05 & 3.77 & $.002 * * *$ & .84 \\
\hline Ansiedad & 2.39 & .34 & .09 & 3.31 & $.005 * * *$ & .58 \\
\hline Depresión & .63 & .53 & .05 & 3.33 & $.005 * * *$ & .74 \\
\hline Creencias desadap. & 1.24 & .23 & .16 & 2.28 & $.037 * * * *$ & .44 \\
\hline Presiones & 1.34 & .20 & .21 & 2.72 & $.016 * * * *$ & .53 \\
\hline Desmotivación & .77 & .45 & .11 & 4.23 & $.001 *$ & .98 \\
\hline Mal afrontamiento & .57 & .34 & .08 & 3.89 & $.002 * *$ & .82 \\
\hline
\end{tabular}

Nota: $* p<.001 ; * * p<.005 ; * * * p=.005 ; * * * * p<.05$

baja laboral $(z=3.68, p<.005)$ y nivel de estrés docente $(z=3.77, p=.005)$, así como en todas las dimensiones relativas a estrés docente. De forma ordenada, la intervención produjo, de mayor a menor efecto: primero en la dimensión desmotivación $(z=4.23, p<.001)$, después en la dimensión mal afrontamiento $(z=3.89, p<.005)$, le siguen las dimensiones depresión $(z=3.33, p=.005)$ y ansiedad $(z=3.31, p=.005)$, y finalmente, la dimensión creencias desadaptativas $(z=2.28, p<.05)$.

Para valorar la magnitud del cambio una vez finalizada la intervención, se empleó la $d$ de Cohen (1988). Valores superiores a .8 indican cambios muy importantes, entre .5 y .8 importantes, por debajo de .5 medios, y menores de .2 bajos. En la Tabla 4 se observa como las puntuaciones de la $d$ de Cohen en el grupo control, muestran la existencia de cambios bajos o muy bajos, con valores entre .05 y .23 , mientras que en el grupo experimental alcanza niveles próximos a 1 en las variables días de baja laboral ( $d$ $=.84)$ y estrés docente $(d=.84)$, y en las dimensiones desmotivación $(d=.98)$ y mal afrontamiento $(d$ $=.82$ ), lo que indica que se producen cambios muy importantes en dichas variables tras la aplicación del programa. Asimismo, se producen cambios importantes en las variables depresión $(d=.74)$, ansiedad $(d=.58)$ y presiones $(d=.53)$, y cambios de nivel medio en la variable creencias desadaptativas $(d=$ .44).

Para realizar un análisis individualizado, se calculó para cada uno de los sujetos, tanto del grupo control como del experimental, el porcentaje de cambio entre las puntuaciones postest y pretest, y la variación en percentiles entre ambas puntuaciones.
También se analizó el cambio de una categoría a otra del nivel de estrés docente, teniendo en cuenta para ello los criterios establecidos en el cuestionario. Respecto al porcentaje de cambio en los participantes del grupo control, en la Tabla 5 se observa que dicho porcentaje oscila entre el -5 y el $7 \%$, lo que indicaría unos porcentajes de cambio muy bajos entre las puntuaciones postest y pretest en la variable estrés docente. Por el contrario, en el grupo experimental, excepto los participantes 12 y 14, que muestran un incremento del 33 y del $1 \%$, respectivamente, en sus niveles de estrés docente, todos los participantes muestran reducciones en dicha puntuación, siendo superiores al $20 \%$ en los participantes 2, 3, 4, 5, 6, 9, 13 y 15 (véase Tabla 5).

$\mathrm{Al}$ analizar el cambio de categoría en función de la puntuación total de estrés docente en el grupo control, se observa como a excepción del P7 que pasa de un nivel normal de estrés docente en la puntuación pretest a un nivel bajo de estrés docente en la puntuación postest, el resto de los participantes no muestran variaciones en la categoría asignada en la puntuación pretest y la asignada en la puntuación postest. Respecto al grupo experimental, los participantes 2, 3, 5 y 15 pasan de tener un nivel de estrés docente alto en la fase pretest a tener un nivel de estrés docente normal en la fase postest; el P9 pasa de un nivel de estrés alto a un nivel de estrés moderado; el P7 de moderado a normal; los participantes 6, 10 y 13 pasan de un nivel de estrés normal a muy bajo; el P1 de normal a bajo; el P4 de bajo a muy bajo; los participantes $8,11,14$ y 16 no muestran cambio de categoría entre su puntuación pretest y postest; y por último, el P12, que es el único sujeto 
Tabla 5. Nivel de estrés docente, porcentaje de cambio y categoría de la Escala de Estrés Docente (ED-6) en pretest y postest, de cada uno de los participantes del grupo control y experimental

\begin{tabular}{rrrrrrr}
\hline & \multicolumn{3}{c}{ Control } & \multicolumn{3}{c}{ Experimental } \\
\hline & \multicolumn{2}{c}{ NED } & $\%$ & \multicolumn{2}{c}{ NED } & $\%$ \\
Participante & Pre & Post & & Pre & Post \\
\hline 1 & $237^{\mathrm{e}}$ & $224^{\mathrm{e}}$ & -5 & $187^{\mathrm{c}}$ & $151^{\mathrm{b}}$ & -19 \\
2 & $174^{\mathrm{c}}$ & $179^{\mathrm{c}}$ & 3 & $243^{\mathrm{e}}$ & $176^{\mathrm{c}}$ & -27 \\
3 & $204^{\mathrm{d}}$ & $203^{\mathrm{d}}$ & -1 & $243^{\mathrm{e}}$ & $172^{\mathrm{c}}$ & -32 \\
4 & $133^{\mathrm{a}}$ & $143^{\mathrm{a}}$ & 7 & $164^{\mathrm{b}}$ & $122^{\mathrm{a}}$ & -26 \\
5 & $167^{\mathrm{c}}$ & $169^{\mathrm{c}}$ & 2 & $250^{\mathrm{e}}$ & $190^{\mathrm{c}}$ & -24 \\
6 & $202^{\mathrm{d}}$ & $196^{\mathrm{d}}$ & -3 & $175^{\mathrm{c}}$ & $137^{\mathrm{a}}$ & -22 \\
7 & $170^{\mathrm{c}}$ & $163^{\mathrm{b}}$ & -4 & $191^{\mathrm{d}}$ & $184^{\mathrm{c}}$ & -3 \\
8 & $232^{\mathrm{e}}$ & $222^{\mathrm{e}}$ & -4 & $136^{\mathrm{a}}$ & $125^{\mathrm{a}}$ & -8 \\
9 & $177^{\mathrm{c}}$ & $168^{\mathrm{c}}$ & -5 & $267^{\mathrm{e}}$ & $203^{\mathrm{d}}$ & -24 \\
10 & $244^{\mathrm{e}}$ & $249^{\mathrm{e}}$ & 2 & $182^{\mathrm{c}}$ & $145^{\mathrm{a}}$ & -20 \\
11 & $174^{\mathrm{c}}$ & $171^{\mathrm{c}}$ & -2 & $139^{\mathrm{a}}$ & $135^{\mathrm{a}}$ & -3 \\
12 & $134^{\mathrm{a}}$ & $133^{\mathrm{a}}$ & -1 & $141^{\mathrm{a}}$ & $187^{\mathrm{c}}$ & 33 \\
13 & $149^{\mathrm{b}}$ & $150^{\mathrm{b}}$ & 1 & $174^{\mathrm{c}}$ & $132^{\mathrm{a}}$ & -24 \\
14 & $168^{\mathrm{c}}$ & $169^{\mathrm{c}}$ & 1 & $143^{\mathrm{a}}$ & $144^{\mathrm{a}}$ & 1 \\
15 & $191^{\mathrm{d}}$ & $201^{\mathrm{d}}$ & 5 & $224^{\mathrm{e}}$ & $179^{\mathrm{c}}$ & -25 \\
16 & & & & $155^{\mathrm{b}}$ & $153^{\mathrm{b}}$ & -1 \\
\hline
\end{tabular}

Nota: NED = nivel de estrés docente; $\%$ = porcentaje de cambio; $\mathrm{a}=\mathrm{NED}$ muy bajo; $\mathrm{b}=$ NED bajo $; \mathrm{c}=\mathrm{NED}$ normal $; \mathrm{d}=$ NED moderado; $\mathrm{e}=\mathrm{NED}$ alto

en donde se observa un cambio que muestra un incremento del nivel de estrés docente, pasa de un nivel muy bajo en la fase pretest a un nivel de estrés docente normal en la fase postest (véase Tabla 5).

Por último, al analizar la variación en percentiles entre las puntuaciones pretest y postest de estrés docente para cada uno de los participantes, se observa como en el grupo control tan sólo aparecen variaciones significativas en los participantes 4 y 7 , quienes ven incrementado su percentil un 100 y un $85 \%$ respectivamente. En el resto de los participantes las variaciones en percentiles están por debajo del $20 \%$. Por lo que respecta al grupo experimental, sólo los participantes 12 y 14 presentan un incremento de sus percentiles, concretamente un 28 y $6 \%$, respectivamente, mientras que en los participantes $1,2,3,4,5$, $6,8,9,10,11,13$ y 15 las reducciones en percentiles oscilan entre el 42\% (P15) y el $87 \%$ (P4). En el resto de los participantes las variaciones son inferiores al 20\% (véase Tabla 6).

\section{Discusión}

Una vez analizados los resultados del presente estudio, podemos concluir que se confirma la hipóte-
Tabla 6. Percentil pretest y postest de la Escala de Estrés Docente (ED6) de cada uno de los participantes del grupo control y experimental, y porcentaje de cambio entre percentiles

\begin{tabular}{rrrrrrr}
\hline & \multicolumn{3}{c}{ Control } & \multicolumn{4}{c}{ Experimental } \\
& \multicolumn{2}{c}{ Percentil ED } & $\%$ & \multicolumn{2}{c}{ Percentil ED } & $\%$ \\
Participante & Pre & Post & & Pre & Post \\
& & & & & \\
1 & 94 & 87 & -7 & 60 & 27 & -55 \\
2 & 48 & 46 & -4 & 95 & 48 & -49 \\
3 & 75 & 74 & -1 & 95 & 47 & -50 \\
4 & 8 & 16 & 100 & 40 & 5 & -87 \\
5 & 45 & 46 & 1 & 95 & 61 & -36 \\
6 & 74 & 68 & -8 & 48 & 10 & -79 \\
7 & 46 & 85 & 85 & 63 & 55 & -13 \\
8 & 91 & 85 & -6 & 10 & 5 & -50 \\
9 & 50 & 45 & -10 & 95 & 75 & -21 \\
10 & 95 & 95 & 0 & 53 & 18 & -60 \\
11 & 48 & 46 & -4 & 10 & 8 & -20 \\
12 & 8 & 8 & 0 & 15 & 57 & 28 \\
13 & 25 & 25 & 0 & 48 & 7 & -85 \\
14 & 45 & 45 & 0 & 16 & 17 & 6 \\
15 & 62 & 73 & 18 & 87 & 50 & -42 \\
16 & & & & 30 & 28 & -7 \\
\hline
\end{tabular}

sis de partida que nos planteamos con la presente investigación, ya que se obtuvo una reducción significativa tanto en los niveles de estrés docente, así como en el número de días de baja laboral en el grupo experimental en comparación con el grupo control.

Los análisis realizados muestran que antes de iniciar el entrenamiento en meditación no se encontraron diferencias significativas de partida entre las puntuaciones del grupo control y experimental en ninguna de las variables examinadas. Sin embargo, una vez finalizada la intervención, al comparar las puntuaciones pretest con las postest entre ambos grupos, se ha podido comprobar una reducción significativa en las variables días de baja laboral y estrés docente, así como en tres de las dimensiones relativas a estrés docente (presiones, desmotivación y mal afrontamiento). Posteriormente, al comparar las puntuaciones pretest con las postest en cada uno de los grupos, no se observa ninguna reducción en ninguna de las variables en el grupo control. Sin embargo, en el grupo experimental se observa una reducción significativa en la medida postest con respecto a la pretest en las variables días de baja laboral y estrés docente, así como en todas las dimensiones relativas a estrés docente (ansiedad, depresión, creencias desadaptativas, presiones, desmotivación y mal afrontamiento). Estos resultados concuerdan con los encontrados en otras investigaciones, donde 
se avala la eficacia de las técnicas de mindfulness para la reducción del estrés (Carlson, Speca y Patel, 2003, 2004; Cohen-Katz et al., 2005; Maclean et al., 1997; Solberg, Halvorsen, Sundgot-Borgen, Ingjer y Holen, 1995; Solberg et al., 2000; Westlund, 1993).

Al realizar el análisis individualizado de las diferencias entre las puntuaciones pretest con las postest, teniendo en cuenta el cambio de categoría de la Escala de Estrés Docente (ED-6), se observó una reducción de los niveles de estrés docente en los participantes del grupo experimental excepto en el P12, el cual experimentó un incremento en su nivel de estrés docente, pasando de un nivel muy bajo en la fase pretest a un nivel normal en la fase postest. Este resultado contradictorio, de aumento del nivel de estrés en el P12, podría estar relacionado con el denominado efecto paradójico de ansiedad derivado de la práctica de técnicas de relajación y/o meditación (véase Heide y Borkovec, 1983). Es decir, la práctica de alguna de las técnicas que incluye el programa de mindfulness Meditación Fuir -conciencia de la respiración, repetición de un mantra, conciencia del cuerpo (body-scan), etc.-, podría haber producido en el P12 incrementos en sus niveles de tensión o ansiedad en lugar de reducirlos, por lo que el nivel de estrés docente de este participante podría haber aumentado debido a la intervención. No obstante, es posible que otras variables pudieran ser las responsables o estar implicadas de algún modo, como por ejemplo, la aparición de factores estresantes o determinados acontecimientos significativos en la vida de los participantes. Por ello, una limitación de este estudio es el no haber evaluado la posible aparición de estas variables, antes y durante la aplicación del programa.

A la luz de los resultados obtenidos, puede afirmarse que el programa de entrenamiento en mindfulness empleado en la presente investigación, es eficaz para reducir los niveles de estrés, específicamente los niveles de estrés docente en profesores de ESO, y consecuentemente, para disminuir el número de días de baja laboral por enfermedad en este colectivo (para otras aplicaciones del programa véase p.ej., De la Fuente, Franco y Mañas, 2010; Franco, 2009b; Franco, De la Fuente y Salvador, 2011; Franco, Mañas, Cangas y Gallego, 2010;
Franco, Mañas, Cangas, Moreno y Gallego, 2010; Franco y Navas, 2009). El análisis de los resultados muestra que los efectos producidos por el programa de entrenamiento fueron considerablemente significativos. No obstante, los resultados de esta investigación deben ser considerados con cautela, ya que la muestra del estudio es relativamente pequeña, por lo que se hace explícita la necesidad de replicar este estudio con muestras más amplias.

Por otro lado, no se puede descartar la influencia de factores inespecíficos sobre los resultados obtenidos, como la atención, el apoyo social o la esperanza positiva, de modo que en futuras investigaciones se podría evaluar la presencia o ausencia de éstas y otras variables; como por ejemplo, el grado de familiarización o historia previa con la meditación, la práctica previa o conjunta de técnicas de relajación, etc. Otra limitación de este estudio es el no haber evaluado el nivel o habilidad de mindfulness en los participantes, por ejemplo a través de la Mindful Attention and Awareness Scale (Brown y Ryan, 2003) o del Kentucky Inventory of Mindfulness Skills (Baer, Smith y Allen, 2004), y haberla considerado una variable dependiente. De este modo, se podría haber examinado si el programa de entrenamiento produce incrementos en los niveles de mindfulness en estos instrumentos, e incluso, haber establecido relaciones entre esta medida con las demás variables dependientes. Otro aspecto a tener en cuenta en futuros estudios, que no se ha contemplado en éste, sería establecer correlaciones entre el grado de cumplimiento de la práctica, a través de los autorregistros, con las variables dependientes. Otra de las limitaciones a tener en consideración en investigaciones posteriores, sería confirmar si las mejoras o los cambios producidos se mantienen a lo largo del tiempo a través de medidas de seguimiento.

Por otra parte, futuras investigaciones podrían comparar los efectos que producen diferentes programas de entrenamiento en mindfulness entre sí -por ejemplo, el programa Mindfulness Based Stress Reduction (MBSR; Kabat-Zinn, 1990, 2003b) con la Meditación Fluir-, y de este modo conocer qué programas son más eficaces en alcanzar determinados objetivos y/o en mantener los cambios producidos a lo largo del tiempo. Así mismo, al comparar diferentes programas entre sí, sería posible analizar la mag- 
nitud del efecto en una determinada variable, y en su caso, qué dimensiones dentro de esa variable se ven afectadas y cuáles no, con el propósito de establecer relaciones entre los componentes de un programa determinado con los efectos observados. Por ejemplo, el programa MBSR contiene ejercicios de Yoga pero el programa de Meditación Fluir no; y en el programa de Meditación Fuir se utiliza un mantra y en el MBSR no. Por lo que es de esperar que el programa MBSR produzca ciertos efectos propios de la práctica del Yoga que no producirá el programa de Meditación Fluir, y que la Meditación Fluir produzca ciertos efectos propios del empleo de un mantra que no producirá el programa MBSR. Por último, futuros estudios podrían analizar los efectos que producen las diferentes técnicas, prácticas y ejercicios de un mismo programa, de forma aislada y conjuntamente, con el propósito de determinar cuáles son lo responsables de los cambios observados y cuáles no, así como los posibles efectos de interacción entre ellos, y de este modo mejorar, perfeccionar y adaptar de forma específica los programas existentes.

Los efectos saludables y preventivos de enfermedad que probablemente haya producido el programa de entrenamiento en mindfulness de este estudio, podrían relacionarse, además de con los beneficios que produce la meditación sobre el organismo relacionados con el estrés indicados en la Introducción, con aquellos que demuestran que durante la meditación la respiración se va haciendo cada vez más lenta y profunda, de forma que el ritmo cardiaco va disminuyendo al tiempo que incrementa su intensidad, por lo que todos los órganos del cuerpo comienzan a beneficiarse al recibir una mejor irrigación sanguínea y, por lo tanto, un mayor aporte de oxígeno, lo que se traduce en un mejor funcionamiento orgánico (Quintero, 2006). Así mismo, se ha señalado que mindfulness está teniendo éxito en clínica porque contribuye al restablecimiento del equilibrio emocional y porque favorece los estados de ánimo positivos y las actitudes de aproximación frente a las de evitación; practicar mindfulness produce una mayor actividad cerebral del hemisferio izquierdo (asimetría prefrontal) la cual se asocia, no sólo a un estado de ánimo más positivo, sino también a una reactividad aumentada a los estímulos emocionales positivos y a una mayor habilidad para afrontar estados de ánimo negativos (véase Simón, 2006).

Por otro lado, se han indicado otros mecanismos o variables responsables de los cambios que se producen con la práctica de mindfulness. Por ejemplo, reduciendo la fusión-cognitiva y desarrollando la flexibilidad cognitiva, abandonando el control de los eventos privados (pensamientos, sentimientos, sensaciones corporales, etc.) y promoviendo la aceptación de los mismos (véase Eifert y Forsyth, 2005; Hayes y Stroshal, 2004; Hayes et al., 1999). La aceptación, la exposición y la autorregulación organísmica son otros de los principales mecanismos conductuales propuestos a la base de la explicación de los efectos de mindfulness (e.g., Mañas, 2009; Vallejo, 2006). Por su parte, Hayes y Wilson (2003) además de contemplar la aceptación, la exposición y la autorregulación han señalado otros procesos como el cambio cognitivo y la relajación. Por último, Coffey y Hartman (2008) han establecido tres mecanismos a través de los cuales mindfulness produce efectos beneficiosos relacionados con el bienestar psicológico: la autorregulación emocional, el desapego y la reducción de la rumiación cognitiva.

Finalmente, deseamos hacer explícito, que los programas de intervención basados en mindfulness no han de ser necesaria y exclusivamente de carácter paliativo, sino que pueden serlo también de carácter preventivo. Actualmente asistimos a una preocupación cada vez mayor por la mejora de la calidad educativa. Los resultados de este estudio podrían contribuir, no sólo a reducir los niveles de estrés docente en profesores de ESO, sino también el número de enfermedades y, consecuentemente, el gasto público que suponen las bajas laborales por enfermedad en nuestro país. Al mismo tiempo, intervenciones de este tipo no solo redundan en beneficios para los docentes, sino que sus efectos se extienden al alumnado, y por lo tanto, al proceso de enseñanza-aprendizaje, incrementándose y mejorándose con ello la calidad de nuestro sistema educativo. 


\section{Extended Summary}

The pressure which teachers undergo provokes physical and psychological pathologies to such an extent that the educative community is paying more attention to this problem which affects the quality of education (Sugai \& Horner, 2001; Troman, 2000; Woods \& Carlyle, 2002), and work stress is connected to $50 \%$ of sick leave (Ponce et al., 2005).

Mindfulness in contemporary psychology has been adopted as an approach for increasing awareness and responding skilfully to mental processes that contribute to emotional distress and maladaptive behaviours (Bishop et al., 2004). The practice of mindfulness teaches one to approach internal experiences with curiosity and acceptance, which allows for intensive self-observation without judgement, elaboration, or attempts to fix or change the experience. Mindfulness has been described as a process of bringing a certain quality of attention to momentby-moment experience (Kabat-Zinn, 1990). Nowadays, numerous studies demonstrate the efficacy and multiple applications of techniques, programmes and psychological therapy based on mindfulness (Arias et al., 2006; Baer, 2003; Chiesa \& Serreti, 2009; Germer et al., 2005).

The hypothesis of this research is that a group of teachers of Secondary School Education who participate in a meditation training program will experience a significant reduction of levels of teachers stress and the of days of sick leave compared to a group of teachers who do not participate in this meditation training.

\section{Method}

\section{Participants}

The sample comprised of 31 teachers of Secondary School Education, 8 men and 23 women, at state schools in the province of Granada (Spain). The teachers ranged in age from 26 to $58(M=$ $38.67 ; D T=1.34$ ). Participants were randomly allocated, 16 in the experimental group and the remaining 15 in the control group.

\section{Materials}

The Teacher Stress Scale (ED-6) (Gutiérrez et al., 2005) which comprises 77 items grouped in six dimensions (anxiety, depression, pressure, nonadaptive beliefs, demotivation and coping poorly) was used to evaluate the level of teacher stress. An overall stress score is found by adding up the scores in the six variables above. Each item is a statement that the teacher must show his agreement on a 5pont Likert-type scale $(1=$ completely disagree to 5 $=$ completely agree).

\section{Design}

To analyze the effects of the mindfulness program (independent variable) on teacher stress and the number of days of sick leave (dependent variables), a group comparison design with pre-test post-test measurement was used on an experimental and a control group.

\section{Procedure}

The first thing we did in this study was to offer teachers of Secondary School Education a course entitled 'Prevention and treatment of stress'. Thirtyone teachers enrolled in the course and were assigned at random to the control group or the experimental group, controlling the sex variable so that there were the same number of men and women in both groups, since women teachers are more affected by teacher stress than men (see Santiago et al., 2008).

Then we proceeded to the pre-test evaluation of all the participants in the study. On the one hand, for measuring levels of teacher stress, they were required to fill out the Teacher Stress Scale (ED-6), and, on the other hand, we also registered the number of days of sick leave during the last academic year (2008/2009). Subjects in the control group were told that there would be two shifts and that they would be in the second shift. 
The meditation program was given in $1 \mathrm{hr} 30 \mathrm{~min}$ sessions per week for 10 weeks. The intervention program consisted of the daily practice of a mindfulness technique called Meditación Fluir (Franco, 2009). The main goal of this technique is not to try to control thoughts or to change them or to replace them with others, but on the contrary, to just leave them alone, and accept any idea that might appear or emerge spontaneously, developing a state of full attention to this mental activity, while being aware that thoughts are transitory and non permanent. The practice of Meditación Fluir consists of repeating a word, or mantra, with a free, open mentality, while directing attention toward the abdomen and noticing how air goes in and out while breathing, but not by trying to change or alter the respiration itself, since only awareness of how it happens naturally and without effort is necessary. Therefore, what is essential when Meditación Fluir is practiced is not the thoughts in themselves, but the awareness of them without evaluating, judging or analyzing them, but just watching how they appear and disappear, and letting them go by.

In each of the 10 sessions, in addition to learning and practicing the Meditación Fluir technique, a variety of Acceptance and Commitment Therapy (Hayes et al., 1999) metaphors and exercise are used along with tales from the Zen Tradition (Deshimaru, 2006) and from Vipassana meditation (Hart, 1994) in order to stress and reinforce the assumption of how the attempt to control annoying and unpleasant private events only makes them chronic and aggravates the psychological discomfort they cause, and therefore, the best thing to do is let them flow freely. Finally, body-scan exercises (Kabat-Zinn, 2003b) were another part of the meditation program learned and practiced throughout all 10 sessions.

The structure of the intervention program sessions was the following: a) participants' comments about their practice of mindfulness exercises during the week (doubts, experiences, set-backs, etc.); b) performing a 10-minute body-scan exercise; c) presenting the different metaphors and exercises corresponding to each session; and, d) practicing mindfulness by paying attention to breathing for 20-30 minutes (see Table 1).

At the end of mindfulness training, the levels of teachers stress and the number of days of sick leave (2009/2010) were evaluated again (post-test evaluation) in both groups under the same conditions and with the same instruments as those used before intervention. When the study was completed, the meditation course was given to the control group.

\section{Results}

Table 2 shows the pre-test and post-test means and standard deviations in the control and experimental groups for the variables studied.

In order to analyse the statistical differences in the pre-test and post-test measures between both groups, we employed the Mann-Whitney Test (independent samples). Statistically significant differences appeared between the groups in both variables: days of sick leave $(z=2.73, p=.01)$ and teachers stress $(z=2.23, p<.05)$; as well in three dimensions of teachers stress: demotivation $(z=$ $2.87, p<.01)$, coping poorly $(z=2.64, p=.01)$, and pressure $(z=2.09, p<.05)$ (see Table 3 ).

With the proposal of analysing the statistical differences between the pre-test and post-test measurements, the Wilconxon Test (related samples) was used. Table 4 shows there are no statistically significant differences between the pre-test and post-test measurement in the control group. However, statistically significant differences were observed in the experimental group in the variables number of days of sick leave $(z=3.68, p<.005)$ and in the level of teachers stress $(z=3.77, p=.005)$; as well in all dimensions of teacher stress: demotivation $(z=4.23$, $p<.001)$, coping poorly $(z=3.89, p<.005)$, depression $(z=3.33, p=.005)$, anxiety $(z=3.31, p=.005)$, and finally, non-adaptive beliefs $(z=2.28, p<.05)$.

To determine the magnitude of change that had occurred in the experimental group upon pre-test and post-test measures, Cohen's $d$ (1988) was used. Over .8 shows very important changes, from .5 to .8 important changes, below .5 medium, and less than .2 few. In Table 4, Cohen's $d$ in the control group indicated very few changes, with values varying from .005 to .23 , while in the experimental group there were very important changes in the variables number days of sick leave $(d=.84)$ and teacher 
stress $(d=.84)$, and in the dimensions demotivation $(d=.98)$, coping poorly $(d=.82)$, important changes in the dimensions depression $(d=.74)$, anxiety $(d=$ $.58)$ and pressure $(d=.53)$, and medium changes in non-adaptive beliefs $(d=.44)$.

Finally, the percentage of change between pre-test and post-test scores of the variable teacher stress was calculated. In the control group, this percentage ranged between -5 and $7 \%$ indicating very small percentage changes between pre-test and post-test scores. However, in the experimental group, with the exception of the participant 12 , all participants showed reductions in levels of teachers stress (see Table 5).

\section{Discussion}

After analyzing the results of the present study, we conclude that the hypothesis this research has been confirmed, considering that a significant reduction in the levels of teacher stress and the number of days of sick leave was observed in the experimental group compared to the control group.

Nowadays, meditation techniques have sufficient scientific guarantee for the educational system to plan the inclusion of these techniques into future teacher educational curricula, to prepare teachers with a series of tools and strategies which will enable them to effectively handle the situations of stress they face in their teaching practice.

\section{Referencias}

Abramowitz, J. S., Tolin, D. F. y Street, G. P. (2001). Paradoxical effects of thought suppression: A meta-analysis of controlled studies. Clinical Psychology Review, 21, 683-703.

Anadón, O. (2005). La formación en estrés para la prevención del síndrome de burnout en el currículo de formación inicial de maestros. Revista Interuniversitaria de Formación del Profesorado, 19, 197-220.

Arias, A. J., Steinberg, K., Banga, A. y Trestman, R. L. (2006). Systematic review of the efficacy of meditation techniques as treatments for medical illness. Journal of Alternative \& Complementary Medicine, 12, 817-832.

Baer, R. (2003). Mindfulness training as a clinical intervention: A conceptual and empirical review. Clinical Psychology: Science and Practice, 10, 125-142.

Baer, R. A., Smith, G. T. y Allen, K. B. (2004). Assessment of mindfulness by self-report: The Kentucky inventory of mindfulness skills. Assessment, 11, 191-206.

Barnes, V. A., Treiber, F. A. y Davis, H. (2001). Impact of transcendental meditation on cardiovascular function at rest and during acute stress in adolescents with high normal blood pressure. Journal of Psychosomatic Research, 51, 597-605.

Benson, H., Malhotra, M. S., Goldman, R. F., Jacobs, G. D. y Hopkins, P. J. (1990). Three case reports of the metabolic and electroencephalographic changes during advanced Buddhist meditation techniques. Behavioral Medicine, 16, 90-95.

Bermejo, L. y Prieto, M. (2005). Creencias irracionales en profesores y su relación con el malestar docente. Clínica y Salud, 16, 45-64.

Bishop, S. R., Lau, M., Shapiro, S., Carlson, L., Anderson, N., Cardomy, J., Segal, Z., Abbey, S., Speca, M., Velting, D. y Devins, G. (2004). Mindfulness: A proposed operational definition. Clinical Psychology: Science and Practice, 10, 230-241.

Bisquerra, R. (2005). La educación emocional en la formación del profesorado. Revista Interuniversitaria de Formación del Profesorado, 19, 95114.

Brown, K. W. y Ryan, R. M. (2003). The benefits of being present: The role of mindfulness in psychological well-being. Journal of Personality and Social Psychology, 84, 822-848.

Brown, K. W., Ryan, R. M. y Creswell, J. D. (2007). Mindfulness: Theoretical foundations and evidence for its salutary effects. Psychological Inquiry, 18, 211-237.

Carlson, L. E., Speca, M. y Patel, K. D. (2003). Mindfulness-based stress reduction in relation to quality of life, mood, symptoms of stress, and immune parameters in breast and prostate cancer outpatients. Psychosomatic Medicine, 65, 571581. 
Carlson, L. E., Speca, M. y Patel, K. D. (2004). Mindfulness-based stress reduction in relation to quality of life, mood, symptoms of stress and levels of cortisol, dehydroepiandrosterone sulphate (DHEAS) and melatonin in breast and prostate cancer outpatients. Pscyhoneuroendocrinology, 29, 448-474.

Cerling, P. y Lee, K. (1997). Treat the body, health the mind. Health, 1, 72-78.

Chiesa, A. y Serretti, A. (2009). Mindfulness-based stress reduction for stress management in healthy people: A review and meta-analysis. The Journal of Alternative and Complementary Medicine, 15, 593-600.

Cockerman, W. C. (2001). Handbook of medical sociology. New York: Prentice-Hall.

Coffey, K. A. y Hartman, M. (2008). Mechanisms of action in the inverse relationship between mindfulness and psychological distress. Complementary Health Practice Review, 13, 79-91.

Cohen, J. (1988). Statistical power analysis for the behavioral sciences. Erlbaum: Hillsdale.

Cohen-Katz, J., Wiley, S. D., Capuano, T., Baker, D. M., Kimmel, S. y Shapiro, S. (2005). The effects of mindfulness-based stress reduction on nurse stress and burnout. Part II: A quantitative and qualitative study. Holistic Nursing Practice, 19, 26-35.

De la Fuente, J., Franco, C. y Mañas, I. (2010). Efectos de un programa de entrenamiento en conciencia plena (mindfulness) en el estado emocional en universitarios. Estudios Sobre Educación, 19, 31-52.

Deshimaru, T. (2006). La práctica del Zen. Barcelona: RBA.

Eifert, G. H. y Forsyth, J. P. (2005). Acceptance and commitment therapy for anxiety disorders: A practitioner's treatment guide to using mindfulness, acceptance, and values-based behaviour change strategies. Oakland, CA: New Harbinger Publications.

Eifert, G. H., Forsyth, J. P., Arch, J., Espejo, N., Keller, M. y Langer, D. (2009). Acceptance and commitment therapy for anxiety disorders: Three case studies exemplifying a unified treatment protocol. Cognitive and Behavioral Practice, 16, 368-385.

Extremera, N. y Fernández-Berrocal, P. (2004). La importancia de desarrollar la inteligencia emocional en el profesorado. Revista Iberoamericana de Educación, 33, 1-9.

Franco, C. (2007). Técnicas de relajación y desarrollo personal. Granada: Cepa.

Franco, C. (2009a). Meditación fluir para serenar el cuerpo y la mente. Madrid: Bubok.

Franco, C. (2009b). Reducción de la percepción de estrés en estudiantes de Magisterio meditante la práctica de la meditación fluir. Apuntes de Psicología, 27, 99-109.

Franco, C., De la Fuente, M. y Salvador, M. (2011). Impacto de un programa de entrenamiento en conciencia plena (mindfulness) en las medidas de crecimiento y autorrealización personal. Psicothema, 23, 58-65.

Franco, C., Mañas, I., Cangas, A. J. y Gallego, J. (2010). The applications of mindfulness with students of secondary school: Results on the academic performance, self-concept and anxiety. En M. D. Lytras, P. Ordonez, A. Ziderman, A. Roulstone, H. Maurer y J. B. Imber (Eds), Knowledge Management, Information Systems, E-Learning, and Sustainability Research: Vol. 111. Comunications in Computer and Information Science (pp. 83-97). Greece: Springer.

Franco, C., Mañas, I., Cangas, A., Moreno, E. y Gallego, J. (2010). Reducing of psychological distress in teachers by a mindfulness tranining programme. The Spanish Journal of Psychology, 13, 655-666.

Franco, C. y Navas, M. (2009). Efectos de un programa de meditación sobre los valores en una muestra de estudiantes universitarios. Electronic Journal of Research in Educational Psychology, 19, 1157-1174.

Germer, C. K., Siegel, R. D. y Fulton, P. R. (2005). Mindfulness and psychotherapy. Nueva York: Guilford Press.

Guerrero, E. (2003). Análisis pormenorizado de los grados de burnout y técnicas de afrontamiento del estrés docente en profesorado universitario. Anales de Psicología, 19, 145-158.

Gresson, J. M. (2009). Mindfulness research update: 2008. Complementary Health Practice Review, 14, 10-18.

Gutiérrez, P., Morán, S. y Sanz, I. (2005). Estrés 
docente: elaboración de la escala ED-6 para su evaluación. Revista Electrónica de Investigación y Evaluación Educativa, 11, 47-61.

Hankey, A. (2006). Studies of advanced stages of meditation in the tibetan Buddhist and Vedic tradition. I: A comparison of general changes. Evidence Based Complement Alternative Medicine, 3, 513-521.

Harrison, E. (2004). Aprender a meditar. Barcelona: Amat.

Hart, W. (1994). La vipassana. El arte de la meditación. Madrid: Luz de Oriente.

Hayes, S. C. y Strosahl, K. D. (2004). A practical guide to acceptance and commitment therapy. New York: Springer-Verlag.

Hayes, S. C., Stroshal, K. D. y Wilson, K. G. (1999). Acceptance and commitment therapy. New York: The Guilford Press.

Hayes, S. C. y Wilson, K. G. (2003). Mindfulness: Method and process. Clinical Pschology: Science and Practice, 10, 161-165.

Heide, F. J. y Borkovec, T. D. (1983). Relaxationinduced anxiety: Paradoxical anxiety enhancement due to relaxation training. Journal of Consulting and Clinical Psychology, 51, 171182.

Holen, A. y Halvor, E. (2007). Acem Meditation: An introductory companion. Oslo: Acem Internacional.

Irving, J. A., Dobkin, P. y Park, J. (2009). Cultivating mindfulness in health care professionals: A review of empirical studies of MindfulnessBased Stress Reduction (MBSR) program. Complementary Therapies in Clinical Practice, 15, 61-66.

Jacobs, G. D., Benson, H. y Friedman, R. (1993). Home-based central nervous system assessment of a system assessment of a multifactor behavioral intervention for chronic sleep-onset insomnia. Behavior Therapy, 24, 159-74.

Kabat-Zinn, J. (1990). Full catastrophe living: Using the wisdom of your body and mind to face stress, pain, and illness. New York: Dell Publishing.

Kabat-Zinn, J. (2003a). Mindfulness-based interventions in context: Past, present, and future. Clinical Psychology: Science and Practice, 10, 144-156.
Kabat-Zinn, J. (2003b). Vivir con plenitud las crisis. Cómo utilizar la sabiduría del cuerpo y la mente para afrontar el estrés, el dolor y la enfermedad. Barcelona: Kairós.

Lyubimov, N. N. (1998). Changes in electroencephalogram and evoked potentials during application of the specific form of physiological training. Human Physiology, 25, 171-180.

Maclean, C. R., Walton, K. G., Wenneberg, S. R., Levitsky, D. K., Mandarino, J. P., Waxiri, R., Hillis, S. L. y Schneider, R. H. (1997). Effects of the transcendental meditation program on adaptive mechanism: Changes in hormone levels and responses to stress after 4 months of practice. Psychoneuroendocrinology, 22, 277-295.

Mañas, I. (2009). Mindfulness (Atención Plena): La meditación en psicología clínica. Gaceta de Psicología, 50, 13-29.

Mañas, I., Luciano, M. C. y Sánchez, L. C. (2008). Beginners practising a basic mindfulness technique: An experimental analysis. Comunicación presentada en la $4^{\text {th }}$ Conference of the European Association for Behaviour Analysis, Madrid, España.

Mañas, I., Sánchez, L. C. y Luciano, M. C. (2008, abril). Efectos producidos por un ejercicio de mindfulness (body-scan): Un estudio piloto. Póster presentado en el VII Congreso de la Sociedad Española de Psicología Experimental, San Sebastián-Donostia, España.

McEwen, R. (2002). The end of stress as we know it. Washington D.C.: Joseph Henry Press.

Miró, M. T. (2006). La atención plena (mindfulness) como intervención clínica para aliviar el sufrimiento y mejorar la convivencia. Revista de Psicoterapia, 17, 31-76.

Molina, T., Gutiérrez, A. G., Hernández, L. y Contreras, C. M. (2008). Estrés psicosocial: Algunos aspectos clínicos y experimentales. Anales de Psicología, 24, 353-360.

Moreno, B., Garrosa, E. y González-Romá, J. L. (2000). La evaluación del estrés y el burnout del profesorado. Revista de Psicología del Trabajo y de las Organizaciones, 16, 331-349.

Moriana, J. A. y Herruzo, J. (2004). Estrés y burnout en profesores. International Journal of Clinical and Health Psychology, 4, 597-621. 
Moriana, J. A. y Herruzco, J. (2005). El síndrome de burnout como predictor de bajas laborales de tipo psiquiátrico. Clínica y Salud, 16, 161172.

Peiró, J. M., Zurriaga, R. y González, V. (2002). Análisis y diagnóstico de las situaciones y experiencias de estrés colectivo en las unidades de trabajo y en las organizaciones de servicios sociales. Revista del Instituto Nacional de Seguridad e Higiene en el Trabajo, 20, 11-21.

Ponce, C. R., Bulnes, M. S., Aliaga, J. M., Atalaya, M. C. y Huertas, R. E. (2005). El síndrome del quemado por estrés laboral asistencial en grupos de docentes universitarios. Revista de Investigación en Psicología, 8, 87-112.

Purdon, C. (1999). Thought suppression and psychopathology. Behaviour Research and Therapy, 37, 1029-1054.

Quintero, D. (2006). Conciencia Zen. Buenos Aires: Alhué.

Rassin, E., Merckelbach, H. y Muris, P. (2000). Paradoxical and less paradoxical effects of thought suppression: A critical review. Clinical Psychology Review, 20, 973-995.

Riquelme, A., Buendía, J. y Rodríguez, M. C. (1993). Estrategias de afrontamiento y apoyo social en personas con estrés económico. Psicothema, 5, 83-89.

Sandín, B. (2003). El estrés: Un análisis basado en el papel de los factores sociales. International Journal of Clinical and Health Psychology, 3, 141-157.

Santiago, M. J., Otero, J. M., Castro, C. y Villardefrancos, E. (2008). Occupational stress in secondary school teachers: Examining the role of student's disruptive behaviour and/or attitudes and the perceived difficulty in conflict management. European Journal of Education and Psychology, 1, 39-50.

Segal, Z. V., Williams, J. M. G. y Teasdale, J. D. (2002). Mindfulness-based cognitive therapy for depression: A new approach to preventing relapse. New York: The Guilford Press.
Shapiro, S. y Schwartz, G. (2000). Handbook of self-regulation. New York: Academic Press.

Simón, V. M. (2006). Mindfulness y neurobiología. Revista de Psicoterapia, 17, 5-30.

Solberg, E., Halvorsen, R., Sundgot-Borgen, J., Ingjer, F. y Holen, A. (1995). A modulator of the immune response to physical stress? A brief report. British Journal of Sports Medicine, 29, 255-257.

Solberg, E. E., Ingjer, F., Holen, A., SundgotBorgen, J., Nilsson, S. y Holme, I. (2000). Stress reactivity to and recovery from a standardised exercise bout: A study of 31 runners practising relaxation techniques. British Journal of Sports Medicine, 34, 268-272.

Solberg, E., Holen, A., Ekeberg, O., Osterud, B., Halvorsen, R. y Sandvik, L. (2004). The effects of long meditation on plasma melatonin and blood serotonin. Medical Science Monitor, 10, 96-101.

Sugai, G. y Horner, R. (2001). School climate and discipline: Going to scale. A framing paper for the national summit. University of Oregon: Center on Positive Behavioral Interventions and Supports.

Thera, H. (2003). El cultivo de la atención plena. México: Pax.

Turner, R. J., Wheaton, B. y Lloyd, D. A. (1995). The epidemiology of social stress. American Sociological Review, 60, 104-125.

Troman, G. (2000). Teachers stress in the low-trust society. British Journal of Sociology of Education, 21, 331-353.

Vallejo, M. A. (2006). Mindfulness. Papeles del Psicólogo, 27, 92-99.

Westlund, P. (1993). Acem meditation beneficial for stressed locomotive engineers. Dyade, 2, 36-51.

Wilson, K. G. y Luciano, M. C. (2002). Terapia de aceptación y compromiso. Un tratamiento conductual orientado a los valores. Madrid: Pirámide.

Woods, P. y Carlyle, D. (2002). Teacher identities under stress: The emotions of separation and renewal. International Studies in Sociology of Education, 12, 169-89.

Manuscrito recibido: 03/08/2010 Revisión recibida: 17/11/2010 Aceptado: 07/03/2011 